\title{
MicroRNA-17-92, a Direct Ap-2 $\alpha$ Transcriptional Target, Modulates T-Box Factor Activity in Orofacial Clefting
}

\author{
Jun Wang ${ }^{1}$, Yan Bai ${ }^{1,2}$, Hong Li ${ }^{3}$, Stephanie B. Greene ${ }^{1}$, Elzbieta Klysik ${ }^{1}$, Wei $\mathrm{Yu}^{4}$, Robert J. Schwartz ${ }^{4,5}$, \\ Trevor J. Williams ${ }^{3}$, James F. Martin ${ }^{1,2,5,6 *}$
}

1 Department of Molecular Physiology and Biophysics, Baylor College of Medicine, Houston, Texas, United States of America, 2 Institute of Biosciences and Technology, Texas A\&M Health Science Center, Houston, Texas, United States of America, 3 Department of Craniofacial Biology, UC Denver, Anschutz Medical Campus, Aurora, Colorado, United States of America, 4 Department of Biology and Biochemistry, University of Houston, Houston, Texas, United States of America, $\mathbf{5}$ Texas Heart Institute, Houston, Texas, United States of America, 6 Program in Developmental Biology, Baylor College of Medicine, Houston, Texas, United States of America

\begin{abstract}
Among the most common human congenital anomalies, cleft lip and palate (CL/P) affects up to 1 in 700 live births. MicroRNA (miR)s are small, non-coding RNAs that repress gene expression post-transcriptionally. The miR-17-92 cluster encodes six miRs that have been implicated in human cancers and heart development. We discovered that miR-17-92 mutant embryos had severe craniofacial phenotypes, including incompletely penetrant CL/P and mandibular hypoplasia. Embryos that were compound mutant for miR-17-92 and the related miR-106b-25 cluster had completely penetrant CL/P. Expression of Tbx 1 and $T b \times 3$, the DiGeorge/velo-cardio-facial (DGS) and Ulnar-mammary syndrome (UMS) disease genes, was expanded in miR-17-92 mutant craniofacial structures. Both $T b \times 1$ and $T b \times 3$ had functional miR seed sequences that mediated gene repression. Analysis of miR-17-92 regulatory regions uncovered conserved and functional $A P-2 \alpha$ recognition elements that directed miR-17-92 expression. Together, our data indicate that miR-17-92 modulates expression of critical T-box transcriptional regulators during midface development and is itself a target of Bmp-signaling and the craniofacial pioneer factor AP-2 $\alpha$. Our data are the first genetic evidence that an individual miR or miR cluster is functionally important in mammalian $\mathrm{CL} / \mathrm{P}$.
\end{abstract}

Citation: Wang J, Bai Y, Li H, Greene SB, Klysik E, et al. (2013) MicroRNA-17-92, a Direct Ap-2 $\alpha$ Transcriptional Target, Modulates T-Box Factor Activity in Orofacial Clefting. PLoS Genet 9(9): e1003785. doi:10.1371/journal.pgen.1003785

Editor: Loydie Anne Jerome-Majewska, McGill University, Canada

Received March 18, 2013; Accepted July 25, 2013; Published September 19, 2013

Copyright: (c) 2013 Wang et al. This is an open-access article distributed under the terms of the Creative Commons Attribution License, which permits unrestricted use, distribution, and reproduction in any medium, provided the original author and source are credited.

Funding: This research was supported by National Institutes of Health Grants: NIH 2R01DE/HD12324-14 (JFM), R01 DE $023177-01$ (JFM) and DE 012728 (TJW), and also was supported by the RNA In Situ Hybridization Core at BCM. We acknowledge Institutional Core Grant \#CA16672 High Resolution Electron Microscopy Facility, UT-MD Anderson Cancer Center, Houston, Texas. The funders had no role in study design, data collection and analysis, decision to publish, or preparation of the manuscript.

Competing Interests: The authors have declared that no competing interests exist.

*E-mail: jfmartin@bcm.edu

\section{Introduction}

The evidence that there is a genetic component underlying CL/ $\mathrm{P}$ is compelling. Analysis of a Danish cohort of $\mathrm{CL} / \mathrm{P}$ cases revealed that relatives of patients with $\mathrm{CL} / \mathrm{P}$ have a higher relative risk for $\mathrm{CL} / \mathrm{P}$ compared to background risk levels. This notion of CL/P heritability is also supported by twin studies [1], [2]. Genome wide association studies (GWAS) and mouse genetics studies have also pointed to genes and genomic regions that are associated with CL/P [3], [4].

MiRs repress gene expression post-transcriptionally by WatsonCrick base pairing to the seed sites in the $3^{\prime}$ UTR of target genes. The miR-17-92 cluster, encoding miR-17, miR-18a, miR-19a, miR$20 a$, miR-19b-1, and miR-92a-1, is within a region on chromosome $13 \mathrm{q}$ that when deletion is associated with CL/P, lung hypoplasia, microphthalmia, microcephaly, and small stature in human patients and has phenotypic similarities to Feingold syndrome [5], [6]. Moreover, miR-17-92 is found in an amplified region associated with small cell lung cancer, as well as in B-cell lymphomas, and is over-expressed in several solid tumor types, including breast, colon, lung, pancreas, and prostate cancers [7].

The mouse embryos with miR-17-92 loss-of-function have smaller body size, microphthalmia, heart defects, and lung hypoplasia [6], [8], [9]. Moreover, the miR-17-92 gain-of-function mutants develop lymphoma, indicating that the mouse is an accurate model for the human syndrome [10]. Unlike the miR-1792 loss-of-function mice, its two homologous clusters, miR-106a363 and miR-106b-25 loss-of-function embryos do not exhibit any gross abnormalities.

Our previous findings indicated that $m i R-17-92$ is directly regulated by Bmp-signaling in heart development [9]. Bmpsignaling deficiency in mice and humans has been shown to cause CL/P and other craniofacial anomalies [11], [12]. Interestingly, miR-17-92 has also been shown to be directly regulated by Myc family transcription factors [7]. Here, we show that miR-17-92 deficiency results in orofacial clefting and that the human disease genes $T b x 1$ and $T b x 3$ are direct targets for $m i R-17-92$. Our findings also reveal that miR-17-92 is a direct target for the master regulator of cranial neural crest development $A P-2 \alpha$.

\section{Results}

miR-17-92 mutant embryos have orofacial clefting

We found that miR-17-92 (miR17-92 $\left.{ }^{\text {null/null }}\right)$ mutant embryos had severe craniofacial defects including $\mathrm{CL} / \mathrm{P}$ and mandibular hypoplasia with notching, revealing that miR-17-92 is a critical regulator of craniofacial development (Figure 1A-H, Figure S1). Moreover, by genetically reducing miR-106b-25 dose on the miR- 


\section{Author Summary}

$\mathrm{CL} / \mathrm{P}$ are very common birth defects in humans. The genetic mechanism underlying $\mathrm{CL} / \mathrm{P}$ pathogenesis is poorly understood. MiRs, small non-coding RNAs that function to post-transcriptionally regulate gene expression, have been identified as pivotal modulators of various developmental events and diseases. To date, there is no individual miR or miR cluster that has been identified as functionally essential in mammalian CL/P. Here, we have discovered that deletion of miR-17-92 cluster in mice results in craniofacial malformations including $C L / P$. Importantly, MIR-17-92 is located on a critical human chromosome region associated with $13 q$ deletion syndrome, a chromosomal disorder that presents with defects including $C L / P$, suggesting the advantages of our animal model to study human disease. Moreover, our work demonstrated that miR-17-92 cluster directly repressed Tbox factors, which have critical functions during craniofacial development. We further showed that miR-17-92 was directly activated by Bmp-signaling and transcription factor AP-2 $\alpha$. Together, our work identified a novel miRmediated transcriptional network underlying $\mathrm{CL} / \mathrm{P}$, providing new insights into craniofacial developmental biology.

17-92 ${ }^{\text {null }}$ background, the clefting phenotype was both more severe and completely penetrant, indicating that there is genetic redundancy between these two miR complexes (Figure $1 \mathrm{C}-\mathrm{F}$, Figure $\mathrm{S} 1 \mathrm{E}-\mathrm{H}$ and Table $\mathrm{S} 1$ ).

In addition to cleft lip and mandible defects, both miR-17-92 mutants and miR-17-92 ${ }^{\text {null }}$; miR-106b-25 $5^{\text {null }}$ compound mutants had cleft secondary palate (Figure 1 B, D, H). Expression of mitotic cell marker phospho-Histone $\mathrm{H} 3$ (pHH3) was greatly reduced in $m i R$ 17-92 mutants, indicating that miR-17-92 is required for normal progenitor cell proliferation during orofacial development (Figure 1I-L, Figure S2). Taken together, these data provide the first genetic evidence that miRs are important regulators of mammalian orofacial development and are involved in CL/P.

\section{miR-17-92 is expressed in craniofacial structures}

We generated a miR-17-92 bacterial artificial chromosome (BAC) transgenic LacZ reporter line to follow the expression of primary (pri)-miR-17-92 (Figure S3A). Three individual transgenic lines showed similar LacZ expression pattern, revealing that primiR-17-92 was expressed in branchial arches and frontonasal process (Figure 2A). LacZ was also detected in the nasal structures, calvarial bones, auricle, periocular mesenchyme, and limb mesenchyme (Figure 2K). Sagittal sections on E11.5 embryos revealed LacZ activity in epithelium and mesenchyme of first branchial arch and frontonasal process (Figure 2I). Coronal sections through E12.5 and E13.5 embryos demonstrated LacZ staining in distal tips of the palatal shelves, the mandibular mesenchyme and mesenchyme of forming frontal bones (Figure 2J, L).

In situ with a pri-miR-17-92 probe revealed similar expression pattern as the transgenic LacZ data (Figure 2E-F). Furthermore, in situ analysis with locked nuclei acid (LNA) probes to detect mature $m i R-17$ and $m i R-92 a$ showed that $m i R-17$ and $m i R-92 a$ were highly expressed in branchial arch and frontonasal process (Figure $2 \mathrm{~B}-$ D, G-H). Unlike miR-17-92, expression of $m i R-106 b-25$ was relatively low (Figure S3B-E).

\section{Tbx genes are repressed by miR-17-92}

Tbx1 gain-of-function causes cleft lip and is a miR-17-92 target in the heart [9], [13]. We evaluated the expression of candidate craniofacial miR-17-92 target genes based on bioinformatics analysis, including Tbx3, Fgf10, Pax9, Shox2 and Osr1, in both miR-17-92 null and conditional knock out mutants using $A P-2 \alpha{ }^{\text {cre }}$ driver [14]. In situ hybridization in miR-17-92 mutants demonstrated up-regulated $T b x 3$ expression in mandible, frontonasalderived structures, tongue, and secondary palate at E13.5 (Figure S4A-F, Figure S5E-F) and in paired maxillary processes and nasal process at E10.0 and E10.5 (Figure 3A-B, Figure S4 G-N). Changes in $\mathrm{Tb} \times 1$ and $\mathrm{Fg} f 10$ expression were not detected at E10.0 likely because the expression changes were not dramatic enough to be detected by in situ hybridization (data not shown). TbxI was expanded primarily in the secondary palate, tongue, and oral ectoderm at E13.5 (Figure 3C-D, Figure S5A-D). In addition, $\mathrm{Fg} f 10$ was expanded in distal mandible and tongue (Figure S5I-L), while ectopic Shox2 expression was observed in distal mandible at E13.5 (Figure S5G-H). Expression of Osr1 was upregulated in the distal mandible and frontonasal structures at E13.5 (Figure S5MP). In contrast, the expression pattern of Pax 9 in miR-17-92 null mutant embryos was unchanged compared to control embryos (data not shown). qRT-PCR experiments also showed upregulation of Tbx1, Tbx3, Fgf10, Shox2 and Osr1 in miR-17-92; miR106b-25 compound mutants at E13.5 (Figure 3E).

To evaluate the expression changes of the above genes, we used a $m i R-17-92$ conditional, cre-activated gain-of-function line (miR$\left.17-92^{O E}\right)$ and the $W n t 1^{\text {cre }}$ driver to activate miR-17-92 in cranial neural crest $(\mathrm{CNC})$ [10], [15]. qRT-PCR analysis from $\mathrm{Wnt}^{\text {cre }}$; miR-17-92 ${ }^{O E}$ orofacial tissue revealed that $\mathrm{Fg} f 10$, Tbx1, Tbx3, Osr 1 and Shox2 were significantly repressed (Figure $3 \mathrm{~F}$ ), while there was no obvious morphological defect detected in miR-17-92 overexpression mutants potentially due to moderaterepression of the $m i R$ 17-92 target genes.

\section{Direct regulation of craniofacial development genes by miR-17-92}

Target genes that are repressed by miR-17-92 have a mixture of $m i R-17 / 20 a / 106 b$ and $m i R-92 a / 25$ family seed sites in their $3^{\prime}$ UTRs (Figure S6). Bioinformatics analysis revealed conserved miR-17/20a/ $106 b$ family seed sequence in the $3^{\prime}$ UTR of Fgf10, Shox2 and Osr 1 (Figure S6A-C, F). The Tbx3 3' UTR contained both a miR-17/20a/ $106 b$ family seed site and a miR-92a/25 family seed site (Figure S6DE). We cloned the 3' UTRs of Fgf10, Shox2, Tbx3 and Osr 1 into luc reporter plasmids to test $\mathrm{miR}$ seed sequence function in vitro. Transfections with miR mimics of miR-17-92 resulted in drastic reduction in luciferase activity for all of the reporter plasmids (Figure 3G, Figure S7). Mutation of the respective miR seed sequences within $3^{\prime}$ UTRs of those genes ablated the inhibition by the corresponding miR (Figure 3G, Figure S7). These data suggest that miR-17-92 directly inhibits Fgf10, Shox2, Tbx3 and Osr1.

\section{Bmps regulate miR-17-92 complex in craniofacial development and miR-17-92 overexpression suppresses orofacial clefting in Bmp mutant mice}

Previous work showed that conditional inactivation of Bmprla, $B m p 4$, and Bmp2;Bmp4 in developing facial processes using the Nestin ${ }^{\text {cre }}$ transgenic driver result in orofacial clefting ([11] and Figure S8). This cre driver directs cre activity in facial prominences [11]. Moreover, miR-17-92 is a direct target for Bmp-signaling in cardiac progenitors [9]. We crossed the miR-17$92^{O E}$ line into Nestin ${ }^{\text {cre }}$, Bmp4, Bmp7 conditional mutant background to test whether miR-17-92 gain-of-function could genetically rescue the defects in Bmp mutants. All Nestin ${ }^{\text {Cre }}$, Bmp4 flox/+,$B m p 7^{\text {flox/ }}+$ embryos (23 out of 23) and embryos without Nestin Cre (29 out of 29) had normal morphology (Figure 4A, Figure 

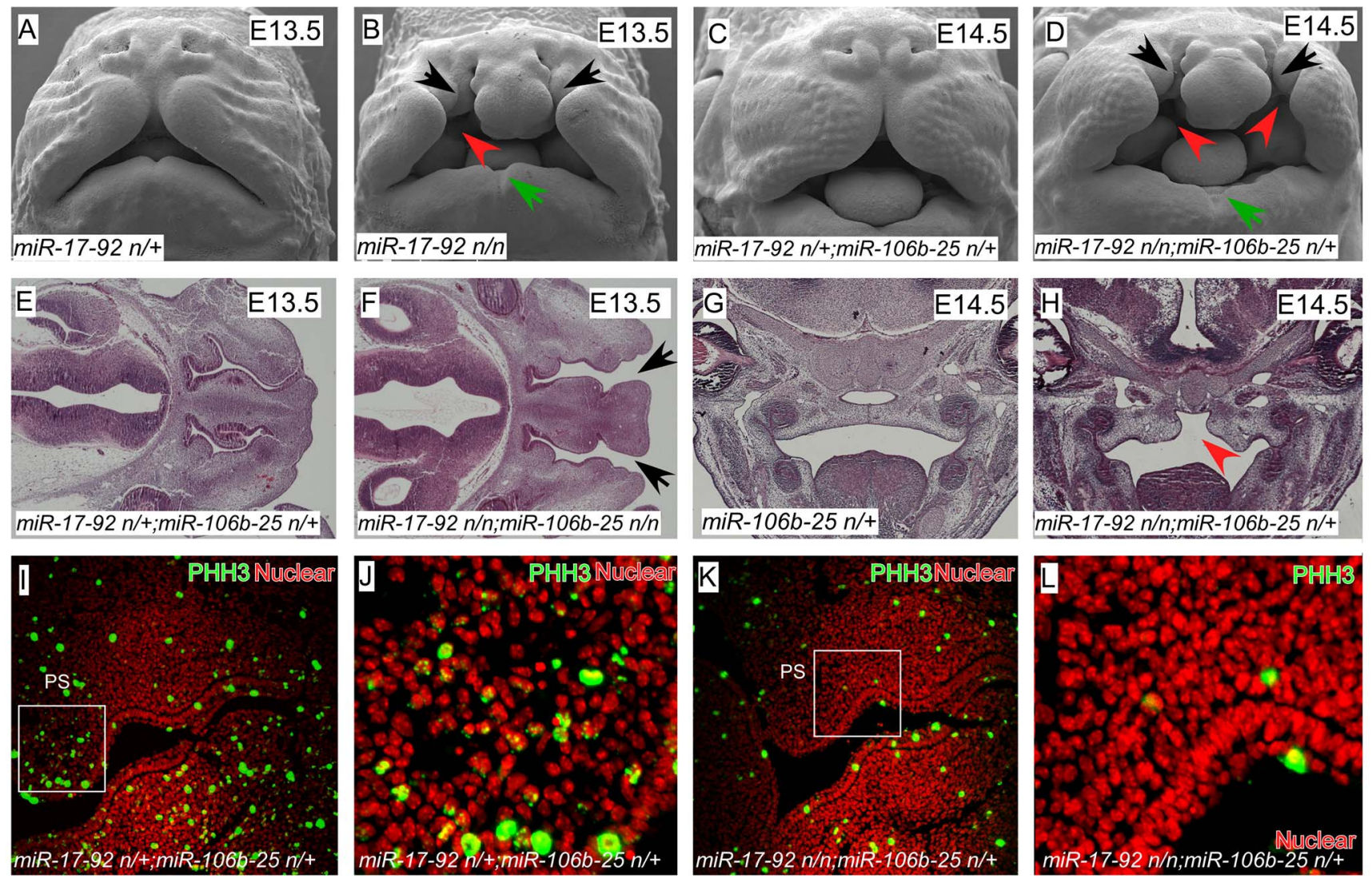

Figure 1. miR-17-92 cluster is required for midface and palate morphogenesis. (A-D) Scanning electron microscopy (SEM) of embryos with the designated genotypes and designated stages (frontal views). miR-17-92 mutants and miR-17-92; miR-106b-25 compound mutants exhibit broad orofacial morphogenesis defects including cleft lip (black arrows), mandibular hypoplasia (green arrows), and cleft palate (red arrowheads). (E-H) Histologic analysis with Hematoxylin-eosin (HE) staining of embryos with labeled genotypes and stages. Transverse sections of E13.5 mouse embryos $(E, F)$ and coronal sections of E14.5 mouse embryos (G, H). Black arrows designate cleft lip and red arrowheads designate cleft palate. (I-L) Immunofluorescence (coronal sections) shows proliferation defects in E12.5 mutant embryos: proliferating cells (green)-stained with PhosphoHiston3 (PHH3); Nuclei (red)-stained with DAPI. doi:10.1371/journal.pgen.1003785.g001

$\mathrm{S} 9 \mathrm{~A}, \mathrm{D}$, table S2), while all Nestin ${ }^{\text {Cre }}, B m p 4^{\text {flox/flox }}, B m p 7^{\text {flox/+ }}$ mutant embryos (6 out of 6 ) had bi-lateral cleft lip and heart defects with incompletely penetrant embryonic lethality at E12.0 likely due to heart defects (Figure 4B, Figure S9B, E, table S2). Most (5 out of 6) Nestin ${ }^{\text {Cre }}, B m p 4^{\text {flox/flox }}, B m p 7^{\text {flox/+ }}, m i R-17-92^{\text {OE }}$ embryos were rescued by $m i R-17-92$ overexpression (significant different compared to Nestin ${ }^{\text {Cre }}, B m p 4^{\text {flox/flox }}, B m p 7^{\text {flox/+ }}$ mutants, CHI-TEST, $\mathrm{p}<0.01$ ), with full suppression of cleft lip and heart defect caused by Bmp loss-of-function, but not eye defect (Figure 4C, Figure S9C, F, table S2).

Consistently, qRT-PCR data indicated that pri-miR-17-92, miR17, and miR-20a were reduced in Bmp2/4 mutant midface (Figure 4D). In situ analysis using miR-17 LNA probe also indicated that $m i R-17$ was dramatically reduced in $B m p 2 / 4$ mutants (Figure S10C, D) compared to controls (Figure S10A, B). In addition, qRT-PGR indicated that $\mathrm{Fg} f 10, \mathrm{~Tb} \times 1, \mathrm{~Tb} \times 3, \mathrm{Osr} 1$ and Shox2 were up-regulated in the midface of $B m p 2 ; B m p 4$ conditional mutants (Figure S10E), further suggesting that these genes are regulated by a BMP-miR-17-92 genetic pathway in craniofacial structures.

Moreover, in vivo chromatin immunoprecipitation (ChIP) data using embryonic midface extracts showed enrichment in the anti-Smad1/5/8 immunoprecipitated chromatin, indicating that Smad1/5/8 directly binds miR-17-92 chromatin (Figure 4E). Co-transfection of a constitutively active Bmprla construct with
miR-17-92 luc reporter resulted in approximately 3-fold induction supporting the hypothesis that Bmp signaling directly regulates $m i R-17-92$ in developing craniofacial structures (Figure 4F). Together, a conserved Bmp-miR-17-92 genetic pathway plays a critical role in the orofacial development.

\section{$A P-2 \alpha$ regulates $m i R-17-92$ in craniofacial development}

Mouse mutants for $A P-2 \alpha$ have $\mathrm{CL} / \mathrm{P}$ and human patients have branchio-oculo-facial syndrome that has $\mathrm{CL} / \mathrm{P}$ as a cardinal feature (BOFS MIM 113620). ChIP-sequencing (ChIP-seq) indicated that $A P-2 \alpha$ bound to miR-17-92 chromatin in cultured human neural crest [16] (Figure 4H, S11A). To determine if $A P-2 \alpha$ directly regulates $m i R-17-92$, we evaluated pri-miR-17-92, mature $m i R-17$, and mature $m i R-20 a$ levels in the $A P-2 \alpha$ mutant midface. qRT-PCR experiments indicated that pri-miR-17-92 and mature miRs were down-regulated in $A P-2 \alpha$ mutants (Figure $4 \mathrm{G}$ ). We used ChIP-PCR to determine whether $A P-2 \alpha$ binds to miR-17-92 chromatin in developing midface tissue. Because there are multiple predicted $A P-2 \alpha$ binding sites in $m i R-17-92$, we subdivided $m i R-17$ 92 into four regions based on ChIP-seq (Figure $4 \mathrm{H}-\mathrm{J}$ and S11). ChIP-PCR experiments using midface extracts indicated that $A P-2 \alpha$ bound to $m i R-17-92$ regions 1 , region 2 , and region 4 (Figure $4 \mathrm{~K}$ ). Transfection experiments with a $m i R-17-92$ reporter containing $A P-2 \alpha$ binding sites revealed that $A P-2 \alpha$ transcription- 

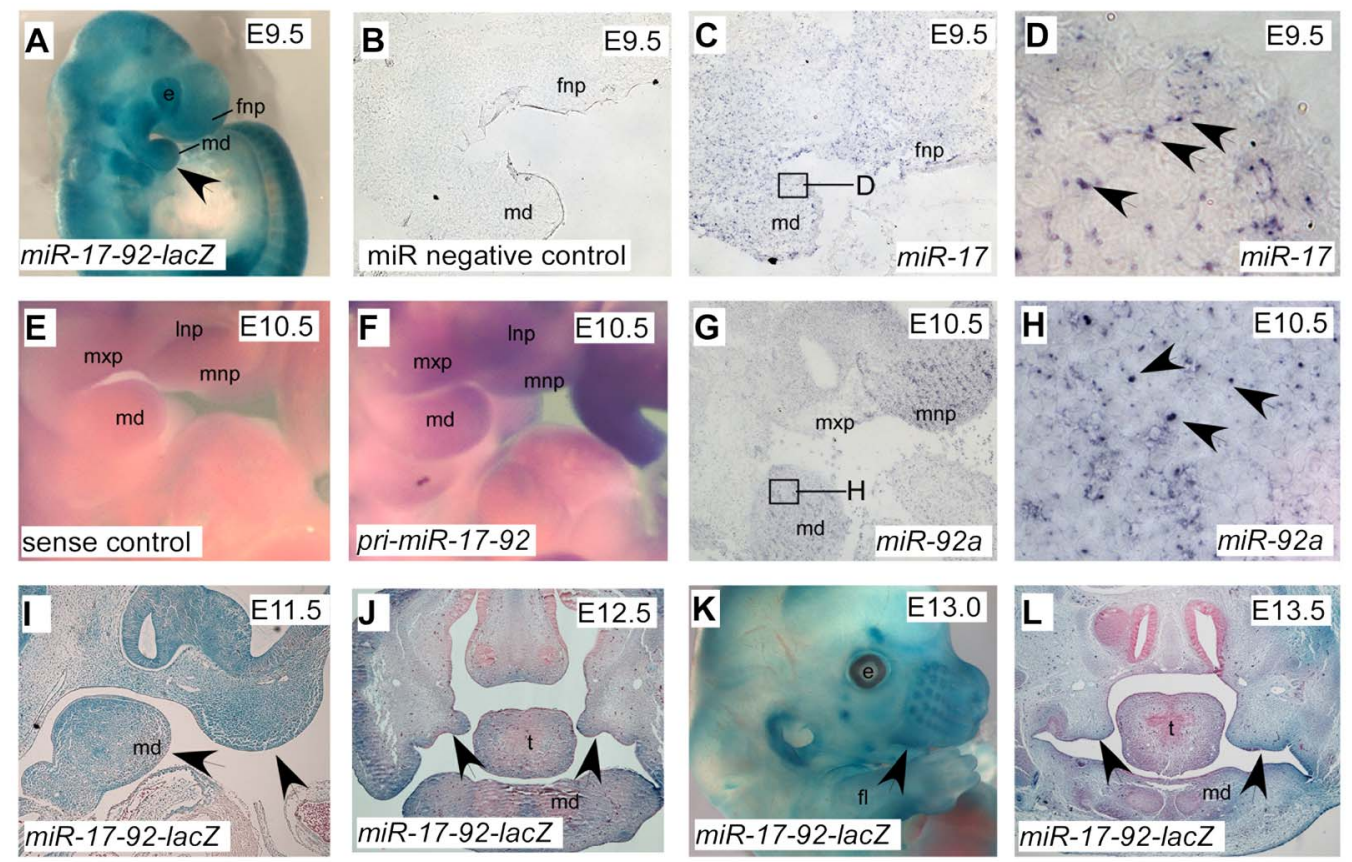

Figure 2. Expression pattern of miR-17-92 at different embryonic stages. $\beta$-Gal stained miR-17-92-lacZ BAC transgenic whole embryo (A), embryonic head $(\mathrm{K})$ and craniofacial sections $(\mathrm{I}, \mathrm{J}, \mathrm{L})$ with the designated stages. Arrowheads indicate positive structures. In situ analysis of pri-miR-17$92(\mathrm{E}, \mathrm{F})$ and mature miR-17 and miR-92a (B-D, G-H) at facial area. Boxed areas in $\mathrm{C}$ and $\mathrm{G}$ are correspondingly shown at higher magnification in $\mathrm{D}$ and $\mathrm{H}$. e, eye; fl, fore-limb; fnp, frontonasal process; $\mathrm{md}$, mandibular process; $\mathrm{mnp}$, medial nasal process; mxp, maxillary process; Inp, lateral nasal process; ns, nose; $t$, tongue.

doi:10.1371/journal.pgen.1003785.g002

ally activated $m i R-17-92$ although synergism with Smadl was not detected using this $m i R-17-92$ reporter (Figure $4 \mathrm{~L}$ ). Moreover, $A P$ $2 \alpha$ may also directly regulate $m i R-106 b-25$ as suggested by the analysis of ChIP-seq data [16] (Figure S12).

\section{Discussion}

We report the first miR underlying mammalian CL/P. miR-1792 is located on human chromosome $13 \mathrm{q} 31.3$ in a critical region for $\mathrm{CL} / \mathrm{P}$ associated with $13 \mathrm{q}$ deletion syndrome highlighting the importance of our findings to human disease. Our data indicated that miR-17-92 promotes proliferation in developing midface by regulating a group of progenitor genes including $T b x 1$ and $T b \times 3$ that are known human disease genes (Figure S13). Our findings reveal that timely down-regulation of progenitor genes in developing midface by miR-17-92 is critical for normal midface development.

\section{Mir-17-92 regulates $T b \times 1$ and $T b \times 3$ genes in craniofacial development}

Tbx1 loss- and gain-of-function result in cleft palate in human DGS patients and mouse models [13], [17]-[19]. Consistent with our findings, Tbx1 gain-of-function results in cell cycle arrest [17]. DGS is characterized by highly variable phenotypes indicating that there are strong modifiers in the human genome [18], [20]. Our findings suggest miR-17-92 as a candidate genetic modifier for $T b x 1$ since it fine-tunes $T b x 1$ expression levels.

Mouse mutants for $T b x 3$ and the related Tbx2 have cleft palate [21]. Furthermore, human patients with UMS have abnormal and distinct facial appearance indicating a requirement for $T b \times 3$ in human craniofacial development [22]. While our findings suggest that elevated $T b x 3$ inhibits proliferation, there is other evidence suggesting that $T b x 3$ promotes proliferation [23]. However, an in vivo study reveals that $T b x 3$ overexpression results in reduced cardiomyocyte proliferation in the zebrafish heart [24]. More work will be required to evaluate $T b \times 3$ function and target genes in vivo in the context of the miR-17-92 mutant midface to better understand contextual $T b \times 3$ function.

\section{MiR-17-92 regulates Fgf signaling}

Both $F g f 10$ and $F g$ fr null mice have cleft secondary palate [25], [26]. Mutations in Fgf10 and Fgf receptors cause lacrimoauricular-dento-digital (LADD) syndrome in human patients indicating a requirement for Fgf-signaling in human craniofacial development [27]. Fgf10 mRNA is enriched in anterior and middle regions of the secondary palate. Moreover, Fgflo deficiency results in abnormal fusion of the palatal to oral cavity epithelium, suggesting that $\mathrm{Fg} f 10$ is required for maturation of palate epithelium. Importantly, elevated Fgf signaling is pathologic in human patients as shown by the extensive investigations into Fgf receptor mediated craniosynostosis [28]. Homozygosity for the Fg fr2 gain-of-function Crouzon mutation in mice results in cleft palate, as well as, craniosynostosis [29] indicating that elevated Fgf signaling also causes cleft palate. Our data demonstrate that $m i R$ 17-92 directly represses $\mathrm{Fg} f 10$ as a mechanism to maintain correct levels of $\mathrm{Fg} f 10$ during palate closure.

\section{Micro RNAs in human orofacial development and disease}

Currently, there are no other genetic loss-of-function data indicating that single miRs or miR clusters are important in mammalian orofacial clefting. Data from zebrafish indicate that miR-140 targets pdgfra to regulate primary palate development [30]. GWAS in human patients reveal important genome regions that are associated with CL/P, including 8q24 [3], [31]. Within the $8 \mathrm{q} 24$ region is the c-myc gene, a known miR-17-92 regulator [32], [33]. Chromosomal deletions that include miR-17-92 cause a 

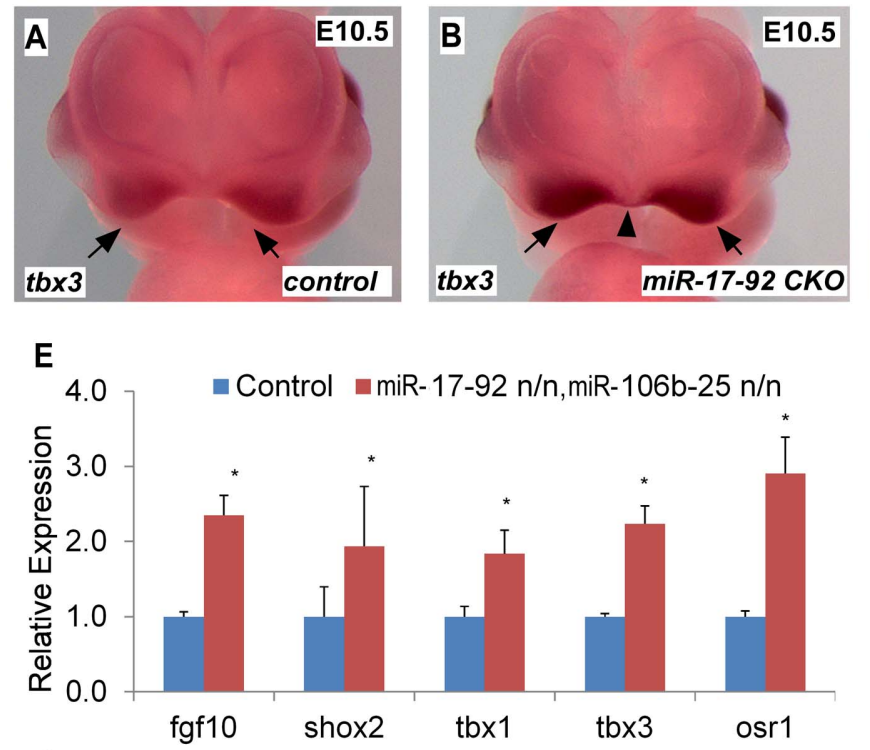

G

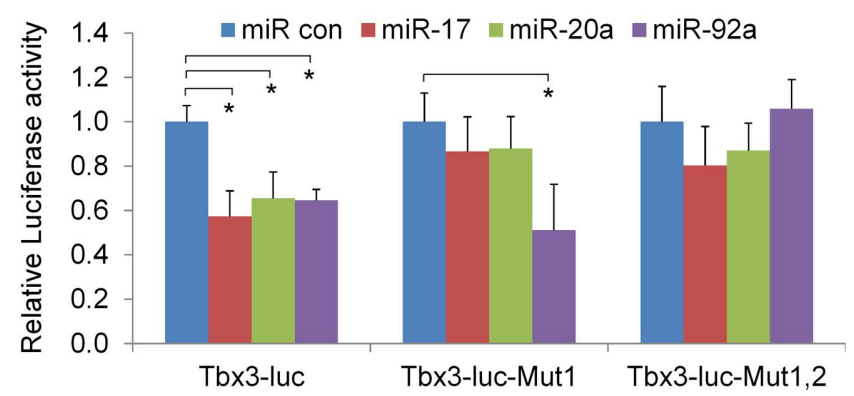

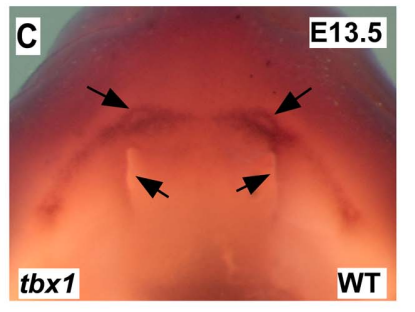

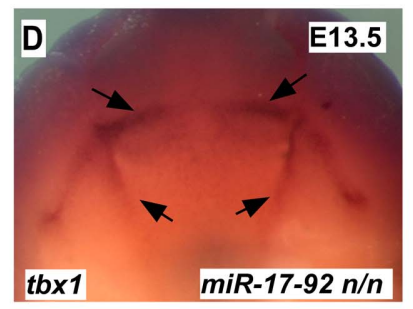

$\mathbf{F}$

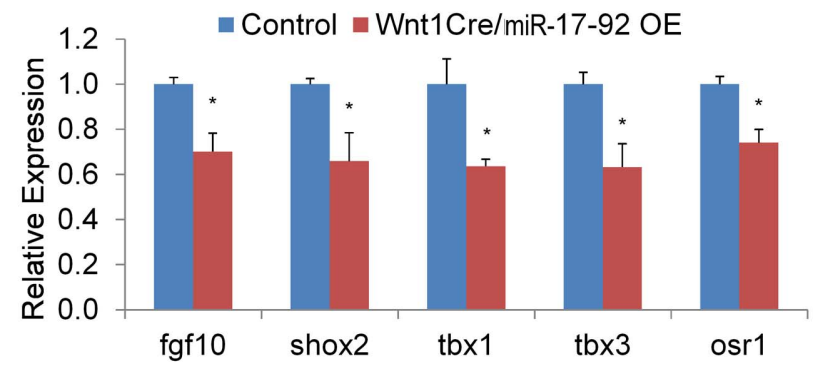

Figure 3. miR-17-92 represses genes important for craniofacial development. (A-D) Whole mount in situ hybridization with indicated probes in mouse embryos with designated genotypes. Black arrows and arrowhead designate expression pattern. (E) qRT-PCR data indicate that expression of Fgf10, Shox2, Tbx1, Tbx3 and Osr1 is elevated in miR-17-92; miR-106b-25 compound knock out mutants. (F) Overexpression of miR-17-92 in cranial neural crest results in repression of Fgf10, Shox2, Tbx1, Tbx3 and Osr1. (G) Luciferase reporter assays with Tbx3 reporters and miRs as labeled (See Fig. S5 for miR seed sites and mutations). mean \pm s.e.m., * indicates statistically significant difference, Student's $t$-test $(P<0.05)$. doi:10.1371/journal.pgen.1003785.g003

variant of Feingold syndrome in human patients with small stature and skeletal abnormalities [6]. Human patients with hemizygous miR-17-92 deletion do not have CL/P likely reflecting phenotypic heterogeneity in miR-17-92 loss of function families. These data are consistent with our findings indicating that there is incomplete penetrance of the CL/P phenotype in miR-17-92 mutant mouse embryos (table S1).

\section{Mir-17-92 regulation in midface development by $\mathrm{Bmp}$} and $A P-2 \alpha$

Consistent with previously finding that Bmp-deficiency results in CL/P in mice and humans [11], [12], our data indicate that Bmp signaling activates miR-17-92 in craniofacial development. Moreover, we show that $A P-2 \alpha$ also regulates miR-17-92 expression although our transfection assays failed to uncover synergistic miR-17-92 activation by $A P-2 \alpha$ and Bmp-signaling (not shown). One possibility is that Bmp-signaling and $A P-2 \alpha$ activate miR-17-92 sequentially during craniofacial progenitor cell development. The assays we employed here cannot easily distinguish molecular events that occur in neighboring or closely apposed cells rather than in the same cell. We also failed to detect up-regulated miR-17-92 target genes in AP-2 $\alpha$ mutants perhaps due to functional redundancy with other AP-2 family members [34]-[36]. Nonetheless our findings have important implications since $A P-2 \alpha$ has been shown to regulate $I f f 6$, a common genetic defect in syndromic and non-syndromic CL/P in human patients [37]. AP-2 $\alpha$ regulation potentially connects $m i R-17-92$ to a gene regulatory network that may be involved in a large portion of human CL/P. In summary, we identified a miR-mediated genetic pathway that plays critical roles during orofacial development (Figure S13).

\section{Materials and Methods}

\section{Ethics statement}

All animal experiments detailed within the manuscript were approved by the Baylor College of Medicine review board.

\section{Mouse alleles and transgenic lines}

The miR-17-92 and miR-106b-25 alleles, Bmp2, Bmp4 and Bmp7 conditional null, $A P-2 \alpha$ cre, Nestin ${ }^{\text {cre }}$ and $W n t t^{\text {cre }}$ alleles were previously described [8], [10], [11], [14], [15], [38]. To generate miR-17-92-lacZ reporter transgenic lines, we obtained the BAC from BACPAC Resources Center, Children's hospital Oakland Research Institute (BAC number: RP23-89P9) and replaced miR17-92 sequence with lacZ coding sequence by recombineering, 

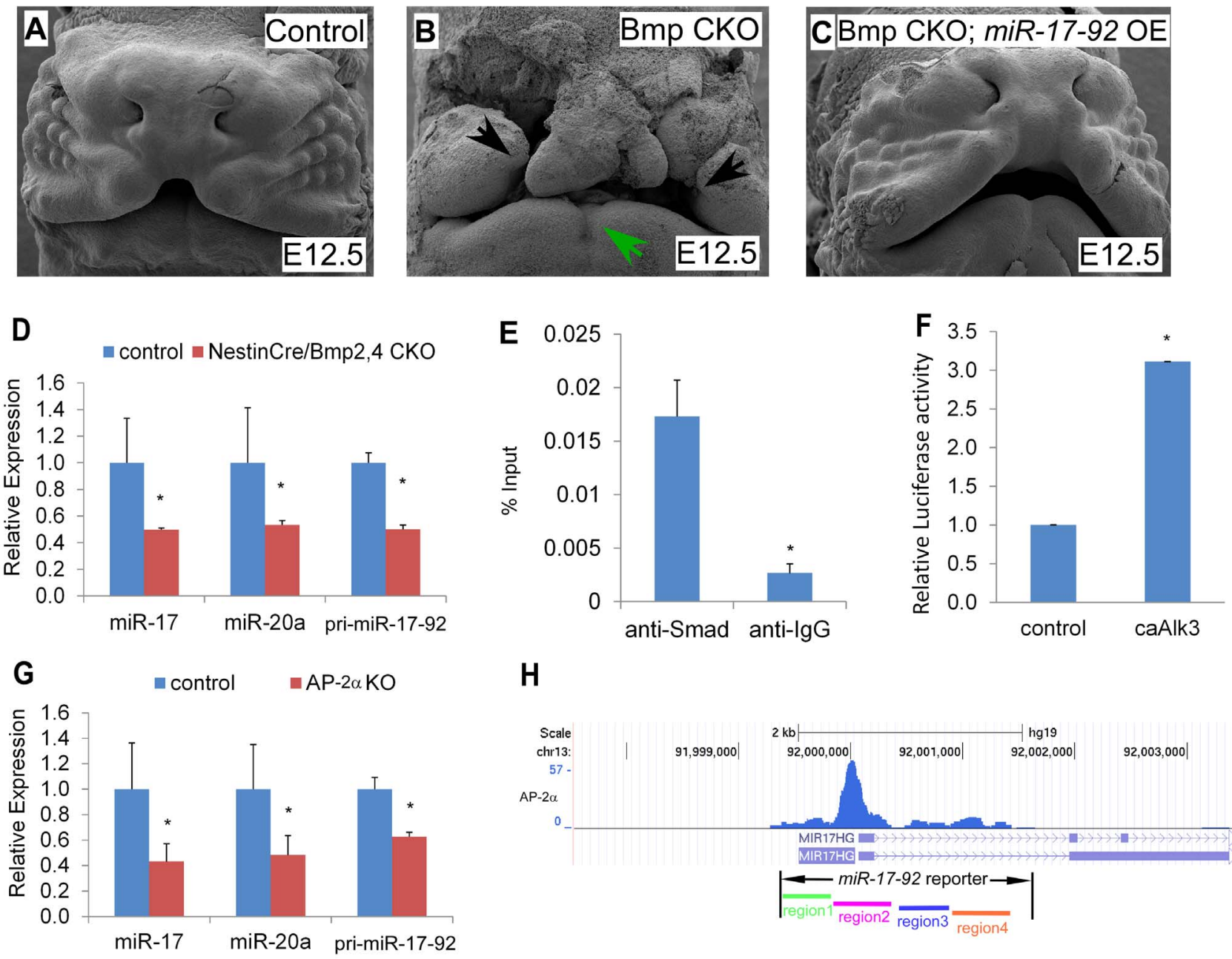

H

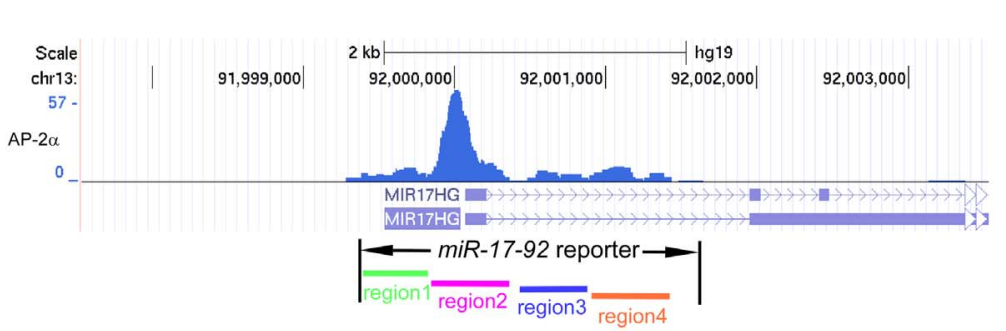

\section{I 遂TCCCCICAGGC \\ AP-2 $\alpha$ :CCCTCAGGG}

\section{J}

Human TTCGCGCCCTGCGGCGAGG 13: 92000020 Mouse TTCGCGCCCTGCGGCGAGG 14:115041049

Human ACCCCCGGCCTGGGGCCTC 13: 92000126 Mouse ACCCCCGGCCTGGGGCCTC 14:115041152
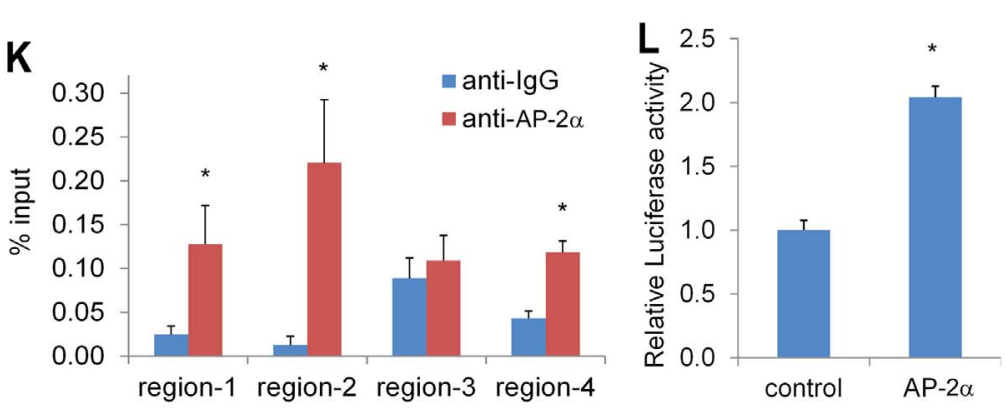

Figure 4. Bmp signals and AP-2 $\alpha$ regulate the miR-17-92 complex in craniofacial structures. (A-C) miR-17-92 overexpression rescue the orofacial cleft in Bmp mutant mice. Scanning electron microscopy (SEM) of embryos with the designated genotypes and designated stages (frontal views). Broad orofacial morphogenesis defects including severe bi-lateral cleft (black arrows) and mandibular hypoplasia (green arrows) are observed in a Nestin ${ }^{\text {Cre }}, B m p 4^{\text {flox/flox }}, B m p 7^{\text {flox/+ }}$ (Bmp CKO) mutant embryo (B) while not in a control (A) and a Nestin ${ }^{\text {Cre }}, B m p 4^{\text {flox/flox }}, B m p 7^{\text {flox/+ }}$, miR-17-92 ${ }^{\text {OE }}$ embryos (Bmp CKO, miR-17-92 OE) (C). (D-G) Bmp signals directly regulate miR17-92. (D) qRT-PCR data indicate that miR-17-92 was reduced in Bmp mutants. (E) In vivo Smad-ChIP-qPCR analysis of the regulatory region in the miR17-92 promoter. (F) miR-17-92 upstream Luc reporter activity cotransfected with constitutively active Alk3 (caAlk3). (G-L) AP-2 $\alpha$ directly regulates miR-17-92. (G) qRT-PCR data indicate that mir-17-92 is reduced in AP-2 $\alpha$ mutants. (H) ChIP-seq peaks indicate AP- $2 \alpha$ binding regions in miR-17-92 chromatin. (I) AP- $2 \alpha$ binding sites information based on overrepresented motifs enriched by ChIP-Seq of neural crest cells [16]. (J) Sequence alignment of two predicted AP-2 $\alpha$ binding sites in region-2 of miR-17-92. (K) In vivo AP-2 $\alpha$ ChIP-qPCR analysis. (L) Co-transfection data of miR-17-92 Luc reporter and AP-2 $\alpha$. mean \pm s.e.m., * indicates statistically significant difference, Student's $t$-test $(P<0.05)$.

doi:10.1371/journal.pgen.1003785.g004

followed by pro-nuclear injection. Constructs were generated using PCR, cloning and recombineering. A LoxP site flanked neo cassette was isolated from PL452 plasmid using BamHI and EcoRI. lacZ coding sequence was isolated from hsp68-lacZ plasmid using BamHI and NcoI. All fragments were cloned into pBluescript SK+ to generate miR-17-92-lac Z construct, followed by recombineering and subsequently Cre mediated recombination for removal of the neo cassette (Figure S3A). 


\section{Scanning Electron Microscopy (SEM)}

Mouse embryos were harvested in ice-cold Phosphate Buffered Saline (PBS), then fixed overnight $(\mathrm{O} / \mathrm{N})$ in $4 \%$ paraformaldehyde (PFA) and $2 \%$ glutaraldehyde in PBS at $4{ }^{\circ} \mathrm{C}$. The samples were then dehydrated in ethanol series to a final $100 \%$ ethanol, followed by transferring to graded series of increasing concentrations of hexamethyldisilazane (HMDS) for $5 \mathrm{~min}$ each and air dried $\mathrm{O} / \mathrm{N}$. Samples were mounted on to double-stick carbon tabs (Ted Pella. Inc.), which have been pre-mounted on to aluminum specimen mounts (Electron Microscopy Sciences). The samples were then coated with a thickness of $25 \mathrm{~nm}$ platinum alloy under vacuum using a Balzer MED 010 evaporator (Technotrade International), then immediately flash carbon coated under the same vacuum. The samples were transferred to a desiccator for later examination. JSM-5910 scanning electron microscope (JEOL, USA, Inc.) was used at an accelerating voltage of $5 \mathrm{kV}$.

\section{Immunofluorescence}

Embryos were fixed in 4\% PFA, embedded in paraffin and cut to $5 \mu \mathrm{m}$ sections mounted on Superfrost/Plus slides (Fisher Scientific). The antigens were retrieved by incubating in the citrate buffer $(10 \mathrm{mM})$ for 2 minutes in microwave oven. The primary antibody was anti-Phospho-Histone H3 with 1:200 dilution (Cell Signaling). Broad HRP conjugated secondary antibody (Invitrogen) was used and visualized using TSA Plus Fluorescence Systems from PerkinElmer on a Zeiss LSM 510 Confocal Microscope. Nuclei were stained with 4,6-diamidino-2-phenylindole (DAPI).

\section{In situ hybridization}

Tissue preparation and in situ hybridization were as previously described [39], [40]. The gene probes were synthesized using DIG RNA Labeling Kit (Roche) following manufacturer's guidelines. The enzymes used for digestion and transcription of in situ constructs are SacII and T7 for Fg f10, XhoI and T7 for Shox2 (gift from Dr. Yiping Chen's lab), EcoRI and T7 for $\operatorname{Osr} 1$ (gift from Dr. Rulang Jiang's lab), EcoRI and T3 for Tbx1(gift from Dr. Antonio Baldini's lab), PstI and T3 for Tbx3 (gift from Dr. Robert Kelly's lab). miRCURY LNA probes were purchased from Exiqon and used per manufacturer's guidelines.

\section{Generation of constructs}

To generate 3' UTR luciferase reporter plasmids, 3' UTR genomic sequence of genes including Fgf10, Osr 1, Shox2 and Tbx3 were amplified using a high-fidelity PCR system (Roche) with designed oligonucleotides and subcloned into the pMIR-REPORT Luciferase miRNA Expression Reporter Vector (Ambion).

Oligonucleotides used to amplify $3^{\prime}$ UTR genomic sequence of $\mathrm{Fg} f 10$ are sense, 5'-CGACTAGTAAGAAAACACTGTTGGTGGATGCAG - $3^{\prime}$, and antisense, 5'-GGAGGGGTTTTTATTCTCTTTTCGCAGG-3'. Oligonucleotides used to amplify $3^{\prime}$ UTR genomic sequence of Osr1 are sense, 5'- GACTAGTATAAACAGAGCCTGCGGG - $3^{\prime}$, and antisense, 5'- CGACGCGTGGCTGTAAAATAACGGTTTATTT -3'. Oligonucleotides used to amplify 3' UTR genomic sequence of Shox2 are sense, 5' -ACTAGTCGCGGGCGCGAGCGCGACGGT-3', and antisense, 5' Oligonucleotides used to amplify $3^{\prime}$ UTR genomic sequence of Tbx3 are sense, 5' - GACTAGTAAACAAGAAAAACAAAATCGCG -3', and antisense, 5'- CGCAAGCTTTCATTTCAATAAAAATTTATTG $-3^{\prime}$. Oligonucleotides used to amplify $3^{\prime}$ UTR genomic sequence of Tbx3 without $\mathrm{mir} 17 /$ mir-20a seed site are sense, 5'- GACTAGTGTGTAACGAGGCTGCTGTTGCTTT - $3^{\prime}$, and antisense, 5' - CGCAAGGTTTGGTCGTTT-
GAACCAAGTCCCTCT - 3 ' . Underlined letters represent enzyme restriction sites for subcloning. All PCR products were sequenced to make sure no mutations were introduced.

\section{Site-directed mutagenesis}

All site-directed mutagenesis of the miR seed sites in the $3^{\prime}$ UTR reporter constructs were achieved by using the QuikChange II site-directed mutagenesis kit (Stratagene). The sense-strand sequences of the oligonucleotides used for mutagenesis (underlined letters indicate the mutation of miR seed sites) were: 5'TAAGACACGGAAGCATTTACTGGAAAGACACTGGGTCATATCATATGCACAACCAAAG- 3' (Fgflo-mut1, $)$, 5'-CCGCATGCGCTCTCAGTTGACTTAATTTGAGACTCTGCAATAAAAAACACCAGCAAT- 3' (Fgf10-mut2), 5'-ACAGCAAATAGTGGAGACGTTGGATTCTTATTTCAACGGGGCATTTAGATTACTAAAGAGA- 3' (Fgf10-mut3); 5'-GCTGACGTTTTTCTGCGAAGTTGAATTCAATAGGAGACATTTGATAAGAG - 3' (Shox2-mut); 5'- GCGGGGCGTTGTATTGGGACTGGGAATTCATGGTGACGATCGGTAACGGAC - 3' (Osr1-mut); 5'- GGACCATTAGTTGTTTTAACTGTATAGAATTCAACAAGGTTTTAAAAGATAATAATA - 3' (Tbx3-mut).

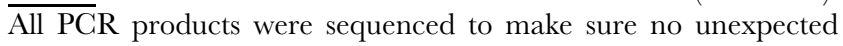
mutations were introduced.

\section{Chromatin immunoprecipitation}

Wild type mouse embryonic orofaces were dissected at E12.5 (for Smad1/5/8 ChIP) or E10.5 (for AP-2 $\alpha \mathrm{ChIP}$ ) and followed by ChIP analysis as previously described [9]. As control, normal rabbit immunoglobulin $\mathrm{G}$ was used as a replacement for the antiSmad1/5/8 (sc-6031 X, Santa Cruz) and 3B5 mouse monoclonal $A P-2 \alpha$ antibody [41] to reveal nonspecific immunoprecipitation of the chromatin. The PCR products were evaluated for appropriate size on a $2 \%$ agarose gel and were confirmed by sequencing. The primers for amplifying the regulatory element in the $5^{\prime}$ upstream of mir-17-92 genomic sequence were: sense, 5'- CTGGCGGGAAGCGTGAGC - 3 ', antisense, 5'-CACGGCGGCTCGTTCTTG -3' (for AP-2 $\alpha$ region 1); sense, 5' - CGTTCATTCACGCACATGGTCGTT - $3^{\prime}$, antisense, 5' - AGCAGCGGCGACGATCTT - $3^{\prime}$ (for AP-2 $\alpha$ region2); sense, 5' - GCACACAATGGCCCTCGG - $3^{\prime}$, antisense, 5' - GCGCGCACAAAGTTTCGG $3^{\prime}$ (for AP-2 $\alpha$ region3); sense, 5' - CGCAGCGGCCCAGAAAC $3^{\prime}$, and antisense, 5' -TCGGCGCGAGCTTATCAAGAGAAA $3^{\prime}$ (for $\mathrm{AP}-2 \alpha$ region4 and Bmp/Smad regulatory element).

\section{Real time RT-PCR}

Total RNA was isolated using RNeasy Micro Kit (QIAGEN) and real-time thermal cycling was performed using StepOne RealTime PCR Systems (Applied Biosystems). Super Script II Reverse Transcriptase (Invitrogen) was used for RT-PCR and SYBR Green JumpStart Taq ReadyMix (SIGMA) was used for real-time thermal cycling. All error bars represent SEM.

\section{Luciferase activity assay}

Plasmids used for transfection were generated as described above or previously reported [9]. LS8 cells were transfected using Lipofectamine 2000 (Invitrogen). Luciferase activity assays were measured using the luciferase Assay System (Promega).

\section{ChIP-seq analysis}

hNCG $A P-2 \alpha$ ChIP-seq and histone modification markers ChIP-seq datasets were accessed from GEO under accession number GSE28876 [16], [42]. Raw fastq reads were mapped to hgl 19 genome using Bowtie2 [43]. The total number of tags of each 
ChIP-seq run was normalized to 10 million. ChIP-seq tracks were visualized and compared in UCSC Genome Browser.

\section{Supporting Information}

Figure S1 miR-17-92 mutant embryos have orofacial clefting. (A-D) miR-17-92 mutant embryos had bilateral cleft lip or unilateral cleft lip (B, D) and cleft palate (not shown) versus their control littermates (A, C). (E-H) Compared with miR-17-92 mutant embryos, miR-17-92 and miR-106b-25 compound mutants had more severe cleft lip phenotypes and more frequent bilateral cleft lip $(F, H)$ versus their control littermates $(E, G)$. (TIF)

Figure S2 Proliferation was reduced in miR-17-92 mutant embryos. (A-H) Immunofluorescence with Phospho-Histon3 (pHH3) antibody (green) at E12.5. Nuclei were stained with DAPI (red). ( $\mathrm{I}-\mathrm{J}$ ) Count of $\mathrm{pHH} 3$ positive cells in epithelial cells (I) and mesenchymal cells $(\mathrm{J})$ in control and miR-17-92 mutant embryos. ps, palate shelf; $t$, tongue.

(TIF)

Figure S3 (A) Schematic diagram of mir-17-92 bacterial artificial chromosome (BAC) transgenic. The LacZ reporter was introduced into a mouse BAC while concurrently the mature miR-17-92 sequences from the BAC were removed. (B-E) In situ hybridization on craniofacial sections indicated expression of miR-106b and $m i R$ 25 in embryonic palate shelf (ps) at E12.5. Black arrows designate signals. Dashed lines show outline of palate shelf.

(TIF)

Figure S4 mir-17-92 represses Tbx3. Whole mount in situ hybridization indicated up-regulation of $T b \times 3$ in $m i R-17-92$ null $(\mathrm{B}, \mathrm{D}, \mathrm{F}, \mathrm{H})$ and $A P-2 \alpha^{C r e}$ conditional knock-out of $m i R-17-92$ (J,L, N) mutants compared to controls (A,C, E, G; I, K, M) at designated stages. Black arrows designate signals. Boxed areas in $\mathrm{K}$ and $\mathrm{L}$ are correspondingly shown at higher magnification in $\mathrm{M}$ and $\mathrm{N}$. (TIF)

Figure S5 mir-17-92 represses genes important for craniofacial development. Whole mount in situ hybridization with indicated probes in mouse embryos with designated genotypes and designated stages. Black arrows designate expressing areas. Embryos were shown in frontal view $(\mathrm{C}, \mathrm{D}, \mathrm{G}, \mathrm{H}, \mathrm{O}, \mathrm{P})$, lateral view $(\mathrm{I}, \mathrm{J}, \mathrm{M}, \mathrm{N})$ and ventral view of roof of mouth and palatal shelves with lower jaw removed (A, B, E, F, K, L). Black arrows designate signals.

(TIF)

Figure S6 Phylogenetic sequence alignment of miR-17-92 family seed sequence in Fgf10 $3^{\prime}$ UTR (A and B), Shox2 3' UTR (C), tbx3 3' UTR (D and E) and Osr13' UTR (F).

(TIF)

Figure S7 mir-17-92 directly regulates Shox2, fgf10 and osr1. (AC) Luciferase reporter assays with reporters and miRs as labeled. Mean \pm s.e.m., * indicates statistically significant difference, Student's $t$-test $(\mathrm{P}<0.05)$.

(TIF)

Figure S8 Cleft lip and palate in Bmp CKO mutants. Genotypes and stages of embryos (A-F) and hematoxylin-eosin (HE) staining sections $(\mathrm{G}-\mathrm{H})$ are as labeled. Dashed lines show outline of midface, arrows designate fusion or clefting, arrowheads designate palate.

(TIF)

Figure S9 $m i R-17-92$ overexpression rescues the orofacial cleft in Bmp CKO mutants. Embryos with the designated genotypes and designated stages are shown in side views $(A-G)$ and frontal views (D-F). A Nestin Cre $, B m p 4^{\text {flox/flox }}, B m p 7^{\text {flox } /+}($ Bmp CKO) mutant embryo $(\mathrm{B}, \mathrm{E})$ had severe bi-lateral cleft, which was observed in in a control (A, C) and a Nestin Cre $, B m p 4^{\text {flox/flox }}, B m p 7^{\text {flox/+ }}, m i R-17$ $92^{O E}$ embryos (Bmp CKO, miR-17-92 OE) (C,F). However, miR17-92 overexpression did not rescue the eye defect in a Bmp CKO mutant $(\mathrm{C})$. Black arrows designate and orofacial structures.

(TIF)

Figure S10 $m i R-17-92$ is a downstream of Bmp signaling. (A-D) In situ analysis of mature miR-17 indicated that mir-17 was dramatically reduced in a Nestin ${ }^{\text {Cre }}, B m p 4^{\text {flox/flox }}, B m p 2^{\text {flox/+ }}(B m p 4$ $-/-, B m p 4+/-\mathrm{CKO})$ mutant $(\mathrm{C}-\mathrm{D})$ compared to a control (A$\mathrm{B})$. Boxed areas in $\mathrm{A}$ and $\mathrm{C}$ are correspondingly shown at higher magnification in B and D. Black arrows designate signals. (E) qRTPCR data indicate that loss of Bmp signals results in elevation of miR-17-92 target genes including Fgf10, Shox2, Tbx1, Tbx3 and Osr 1 . Mean \pm s.e.m., * indicates statistically significant difference, Student's $t$-test $(\mathrm{P}<0.05)$.

(TIF)

Figure S11 AP- $2 \alpha$ directly regulates the miR-17-92 complex. (A) AP- $2 \alpha$ and histone modification markers ChIP-seq data in cultured human neural crest and Hela cells. (B) Sequence alignment of AP-2 $\alpha$ binding region-1, 3 and 4 in miR-17-92. Region 1 contains three potential binding sites, region 3 and 4 contain two. Region two was shown in Figure 4.

(TIF)

Figure S12 The miR-106b-25 complex is a potential AP- $2 \alpha$ target, which is suggested by $\mathrm{AP}-2 \alpha$ and histone modification markers ChIP-seq data in cultured human neural crest and Hela cells.

(TIF)

Figure S13 A model for the Bmp/AP-2 $\alpha-m i R-17-92-T b x$ pathway during orofacial development.

(TIF)

Table S1 Summary of phenotypes of miR-17-92 single and miR17-92; miR-106b-25 compound mutant embryos. For cleft lip and palate, the penetrance and severity of the phenotype was more severe in compound mutants for both miR clusters. A "wide mouth" phenotype that was observed in some miR17-92 mutants represents an increased distance between the two frontonasal processes and we believe this is an intermediate phenotype between normal and cleft lip.

(DOCX)

Table S2 Phenotype summary of embryos with different genotypes. At embryonic day 10.5 to 12.5 (E10.5-E12.5), all Nestin Cre, Bmp4 flox/flox, Bmp7 flox/+ mutant embryos (Nestin Cre, $B 4 \mathrm{f} / \mathrm{f}, B 7 f /+)$ have severe bi-lateral cleft lip and heart defect. In most case, miR-17-92 over expression rescue cleft lip and heart defect caused by Nestin Cre, B4 f/f, B7f/+ but not eye defect. 83.33\% (5 out of 6) Nestin Cre, Bmp4 flox/flox, Bmp7 flox/+, miR-1792-OE (Nestin Cre, B4 f/f, B7f/+, miR-OE) mutant embryo were fully rescued and $16.67 \%$ (1 out of 6 ) had bi-lateral cleft lip and heart defect. Two Nestin Cre, B4 f/f, B7f/+ mutants died at E12.0 likely due to severe heart defect and 7 embryos were not able to genotyped due to early embryonic lethal at E9.5. * miR-17-92 over expression rescued cleft lip (compared to Bmp CKO, CHI-TEST, $\mathrm{p}<0.01)$.

(DOCX)

\section{Acknowledgments}

We thank Min Zhang for help with ChIP-seq analysis. 


\section{Author Contributions}

Conceived and designed the experiments: JW JFM. Performed the experiments: JW YB HL SBG EK WY. Analyzed the data: JW YB HL

\section{References}

1. Grosen D, Bille C, Pedersen JK, Skytthe A, Murray JC, et al. (2010) Recurrence risk for offspring of twins discordant for oral cleft: a population-based cohort study of the Danish 1936-2004 cleft twin cohort. Am J Med Genet A 152A: 2468-2474.

2. Grosen D, Chevrier C, Skytthe A, Bille C, Molsted K, et al. (2010) A cohort study of recurrence patterns among more than 54,000 relatives of oral cleft cases in Denmark: support for the multifactorial threshold model of inheritance. J Med Genet 47: 162-168.

3. Beaty TH, Murray JC, Marazita ML, Munger RG, Ruczinski I, et al. (2010) A genome-wide association study of cleft lip with and without cleft palate identifies risk variants near MAFB and ABCA4. Nat Genet 42: 525-529.

4. Dixon MJ, Marazita ML, Beaty TH, Murray JC (2011) Cleft lip and palate: understanding genetic and environmental influences. Nat Rev Genet 12: 167178.

5. Kirchhoff M, Bisgaard AM, Stoeva R, Dimitrov B, Gillessen-Kaesbach G, et al. (2009) Phenotype and 244k array-CGH characterization of chromosome 13q deletions: an update of the phenotypic map of 13q21.1-qter. Am J Med Genet A 149A: 894-905.

6. de Pontual L, Yao E, Callier P, Faivre L, Drouin V, et al. (2011) Germline deletion of the miR-17-92 cluster causes skeletal and growth defects in humans. Nat Genet 43: 1026-1030.

7. Mendell JT (2008) miRiad roles for the miR-17-92 cluster in development and disease. Cell 133: 217-222.

8. Ventura A, Young AG, Winslow MM, Lintault L, Meissner A, et al. (2008) Targeted deletion reveals essential and overlapping functions of the miR-17 through 92 family of miRNA clusters. Cell 132: 875-886.

9. Wang J, Greene SB, Bonilla-Claudio M, Tao Y, Zhang J, et al. (2010) Bmp signaling regulates myocardial differentiation from cardiac progenitors through a MicroRNA-mediated mechanism. Dev Cell 19: 903-912.

10. Xiao C, Srinivasan L, Calado DP, Patterson HC, Zhang B, et al. (2008) Lymphoproliferative disease and autoimmunity in mice with increased miR-1792 expression in lymphocytes. Nat Immunol 9: 405-414.

11. Liu W, Sun X, Braut A, Mishina Y, Behringer RR, et al. (2005) Distinct functions for Bmp signaling in lip and palate fusion in mice. Development 132: 1453-1461.

12. Suzuki S, Marazita ML, Cooper ME, Miwa N, Hing A, et al. (2009) Mutations in BMP4 are associated with subepithelial, microform, and overt cleft lip. Am J Hum Genet 84: 406-411.

13. Fulcoli FG, Huynh T, Scambler PJ, Baldini A (2009) Tbx1 regulates the BMPSmadl pathway in a transcription independent manner. PLoS One 4: e6049.

14. Macatee TL, Hammond BP, Arenkiel BR, Francis L, Frank DU, et al. (2003) Ablation of specific expression domains reveals discrete functions of ectodermand endoderm-derived FGF8 during cardiovascular and pharyngeal development. Development 130: 6361-6374.

15. Chai Y, Jiang X, Ito Y, Bringas P, Han J, et al. (2000) Fate of the mammalian cranial neural crest during tooth and mandibular morphogenesis. Development 127: $1671-1679$.

16. Rada-Iglesias A, Bajpai R, Prescott S, Brugmann SA, Swigut T, et al. (2012) Epigenomic annotation of enhancers predicts transcriptional regulators of human neural crest. Cell Stem Cell 11: 633-648.

17. Funato N, Nakamura M, Richardson JA, Srivastava D, Yanagisawa H (2012) Tbxl regulates oral epithelial adhesion and palatal development. Hum Mol Genet 21: 2524-2537.

18. Widdershoven JC, Bowser M, Sheridan MB, McDonald-McGinn DM, Zackai $\mathrm{EH}$, et al. (2013) A candidate gene approach to identify modifiers of the palatal phenotype in 22q11.2 deletion syndrome patients. Int J Pediatr Otorhinolaryngol 77: 123-127.

19. Portnoi MF (2009) Microduplication 22q11.2: a new chromosomal syndrome. Eur J Med Genet 52: 88-93.

20. Aggarwal VS, Morrow BE (2008) Genetic modifiers of the physical malformations in velo-cardio-facial syndrome/DiGeorge syndrome. Dev Disabil Res Rev 14: 19-25.

21. Zirzow S, Ludtke TH, Brons JF, Petry M, Christoffels VM, et al. (2009) Expression and requirement of T-box transcription factors Tbx2 and Tbx3 during secondary palate development in the mouse. Dev Biol 336: 145-155.
SBG. Contributed reagents/materials/analysis tools: RJS TJW. Wrote the paper: JW JFM.

22. Joss S, Kini U, Fisher R, Mundlos S, Prescott K, et al. (2011) The face of Ulnar Mammary syndrome? Eur J Med Genet 54: 301-305.

23. Lee JM, Kim JY, Cho KW, Lee MJ, Cho SW, et al. (2007) Modulation of cell proliferation during palatogenesis by the interplay between Tbx3 and Bmp4. Cell Tissue Res 327: 285-292.

24. Ribeiro I, Kawakami Y, Buscher D, Raya A, Rodriguez-Leon J, et al. (2007) Tbx2 and Tbx3 regulate the dynamics of cell proliferation during heart remodeling. PLoS One 2: e398.

25. Alappat SR, Zhang Z, Suzuki K, Zhang X, Liu H, et al. (2005) The cellular and molecular etiology of the cleft secondary palate in Foflo mutant mice. Dev Biol 277: $102-113$.

26. Rice R, Spencer-Dene B, Connor EC, Gritli-Linde A, McMahon AP, et al. (2004) Disruption of Fgf10/Fgfr2b-coordinated epithelial-mesenchymal interactions causes cleft palate. J Clin Invest 113: 1692-1700.

27. Rohmann E, Brunner HG, Kayserili H, Uyguner O, Nurnberg G, et al. (2006) Mutations in different components of FGF signaling in LADD syndrome. Nat Genet 38: 414-417.

28. Chai Y, Maxson RE, Jr. (2006) Recent advances in craniofacial morphogenesis. Dev Dyn 235: 2353-2375.

29. Eswarakumar VP, Ozcan F, Lew ED, Bae JH, Tome F, et al. (2006) Attenuation of signaling pathways stimulated by pathologically activated FGF-receptor 2 mutants prevents craniosynostosis. Proc Natl Acad Sci U S A 103: 18603-18608.

30. Eberhart JK, He X, Swartz ME, Yan YL, Song H, et al. (2008) MicroRNA Mirn140 modulates Pdgf signaling during palatogenesis. Nat Genet 40: 290298.

31. Birnbaum S, Ludwig KU, Reutter H, Herms S, Steffens M, et al. (2009) Key susceptibility locus for nonsyndromic cleft lip with or without cleft palate on chromosome 8q24. Nat Genet 41: 473-477.

32. O'Donnell KA, Wentzel EA, Zeller KI, Dang CV, Mendell JT (2005) c-Mycregulated microRNAs modulate E2F1 expression. Nature 435: 839-843.

33. Dews M, Homayouni A, Yu D, Murphy D, Sevignani C, et al. (2006) Augmentation of tumor angiogenesis by a Myc-activated microRNA cluster. Nat Genet 38: 1060-1065.

34. Winger Q, Huang J, Auman HJ, Lewandoski M, Williams T (2006) Analysis of transcription factor AP-2 expression and function during mouse preimplantation development. Biol Reprod 75: 324-333.

35. Satoda M, Zhao F, Diaz GA, Burn J, Goodship J, et al. (2000) Mutations in TFAP2B cause Char syndrome, a familial form of patent ductus arteriosus. Nat Genet 25: 42-46.

36. Li W, Cornell RA (2007) Redundant activities of Tfap2a and Tfap2c are required for neural crest induction and development of other non-neural ectoderm derivatives in zebrafish embryos. Dev Biol 304: 338-354.

37. Rahimov F, Marazita ML, Visel A, Cooper ME, Hitchler MJ, et al. (2008) Disruption of an AP-2alpha binding site in an IRF6 enhancer is associated with cleft lip. Nat Genet 40: 1341-1347.

38. Bai Y, Wang J, Morikawa Y, Bonilla-Claudio M, Klysik E, et al. (2013) Bmp signaling represses Vegfa to promote outflow tract cushion development. Development 140: 3395-3402.

39. Yaylaoglu MB, Titmus A, Visel A, Alvarez-Bolado G, Thaller C, et al. (2005) Comprehensive expression atlas of fibroblast growth factors and their receptors generated by a novel robotic in situ hybridization platform. Dev Dyn 234: 371386.

40. Lu MF, Pressman C, Dyer R, Johnson RL, Martin JF (1999) Function of Rieger syndrome gene in left-right asymmetry and craniofacial development. Nature 401: 276-278.

41. Turner BC, Zhang J, Gumbs AA, Maher MG, Kaplan L, et al. (1998) Expression of AP-2 transcription factors in human breast cancer correlates with the regulation of multiple growth factor signalling pathways. Cancer Res 58: 5466-5472.

42. Rada-Iglesias A, Bajpai R, Swigut T, Brugmann SA, Flynn RA, et al. (2011) A unique chromatin signature uncovers early developmental enhancers in humans. Nature 470: 279-283.

43. Langmead B, Trapnell C, Pop M, Salzberg SL (2009) Ultrafast and memoryefficient alignment of short DNA sequences to the human genome. Genome Biol 10: R25. 